\title{
Plasmodium infection inhibits the expansion and activation of MDSCs and Tregs in the tumor microenvironment in a murine Lewis lung cancer model
}

Dickson Adah ${ }^{1,2}$, Yijun Yang ${ }^{1,2}$, Quan Liu ${ }^{1,2}$, Kranthi Gadidasu' ${ }^{1}$ Z Zhu Tao ${ }^{1}$, Songlin Yu ${ }^{1,2}$, Linglin Dai ${ }^{1,3}$, Xiaofen Li ${ }^{1,3}$, Siting Zhao ${ }^{1,3}$, Limei Qin ${ }^{1}$, Li Qin ${ }^{1,3^{*}}$ and Xiaoping Chen ${ }^{1,3^{*}}$ (D)

\begin{abstract}
Background: A major challenge in the development of effective cancer immunotherapy is the ability of tumors and their microenvironment to suppress immune cells through immunosuppressive cells such as myeloid -derived suppressor cells and regulatory $T$ cells. We previously demonstrated that Plasmodium infection promotes innate and adaptive immunity against cancer in a murine Lewis lung cancer model but its effects on immunosuppressive cells in the tumor microenvironment are unknown.

Methods: Whole Tumors and tumor-derived sorted cells from tumor-bearing mice treated with or without plasmodium infected red blood cells were harvested 17 days post tumor implantation and analyzed using QPCR, western blotting, flow cytometry, and functional assays. Differences between groups were analyzed for statistical significance using Student's t-test.

Results: Here we found that Plasmodium infection significantly reduced the proportions of MDSCs and Tregs in the lung tumor tissues of the treated mice by downregulating their recruiting molecules and blocking cellular activation pathways. Importantly, $\mathrm{CD}^{+} \mathrm{T}$ cells isolated from the tumors of Plasmodium-treated mice exhibited significantly higher levels of granzyme B and perforin and remarkably lower levels of PD-1.

Conclusion: We reveal for the first time, the effects of Plasmodium infection on the expansion and activation of MDSCs and Tregs with a consequent elevation of $\mathrm{CD}^{+}{ }^{+} \mathrm{T}$ cell-mediated cytotoxicity within the tumor microenvironment and hold great promise for the development of effective immunotherapeutic strategies.
\end{abstract}

Keywords: Lung cancer, MDSC, Tregs, Recruiting molecules, PD-1

\section{Background}

A key pathological feature of solid cancer is the massive mobilization and infiltration of immune cells into the lymphoid organs and the tumor microenvironment. Solid tumors are known for their ability to recruit and modify immune cells and endothelial cells which form the tumor microenvironment [1]. A growing body of evidence suggests that host immune cells with a suppressive

\footnotetext{
* Correspondence: qin_li@cas-lamvac.com; chen_xiaoping@gibh.ac.cn ${ }^{1}$ State Key Laboratory of Respiratory Disease, Center of Infection and Immunity, Guangzhou Institutes of Biomedicine and Health, Chinese Academy of Sciences, 190 Kaiyuan Avenue, Science Park, Guangzhou 510530, People's Republic of China

Full list of author information is available at the end of the article
}

phenotype pose a significant challenge to the success of immune-enhancing anticancer therapy [2-5]. Among these suppressor cells, myeloid-derived suppressor cells (MDSCs) and regulatory T cells (Tregs) have been shown to increase significantly in number in hosts with advanced malignancies [6, 7]. MDSCs are a heterogeneous population of cells that expand during cancer, inflammation and infection, and have a remarkable ability to suppress T-cell responses [8]. There are two main subsets of MDSCs: polymorphonuclear MDSCs (P-MDSCs), which are similar in phenotype and morphology to neutrophils, and monocytic MDSCs (M-MDSCs), which are similar to monocytes [9-11]. In cancer, several differences in 
distribution and functions have been observed in these MDSC subsets. In the peripheral lymphoid organs, there are more P-MDSCs than M-MDSCs; these P-MDSCs have a relatively modest suppressive activity and play a key role in the regulation of tumor-specific immune responses, facilitating the development of tumor-specific T-cell tolerance. M-MDSCs in the peripheral lymphoid organs lack the capacity to differentiate into macrophages (MØ) and dendritic cells (DC). In contrast, MDSCs in the tumor microenvironment are more suppressive, mostly M-MDSC, and can differentiate into tumor- associated macrophages (TAMs)(reviewed in [12]). This further highlights the importance of novel strategies to inhibit multiple subsets of MDSCs both in the peripheral lymphoid organs and in the tumor microenvironment. Several MDSC-inhibiting drugs have been tested but only against splenic MDSCs, highlighting the need for the development of immune-enhancing drugs and interventions to target MDSCs in the tumor microenvironment.

Growing evidence points to the accumulation of P-MDSC and M-MDSC subsets accompanied by a dynamic change in the macrophages that enter the tumor site toward a predominantly IL10-producing M2 phenotype, all of which have the ability to induce T-regs cells and/or directly suppress antitumor $\mathrm{T}$ cell function [13]. In tumor-bearing mice, MDSCs can themselves facilitate the generation of tumor-specific Tregs [14] suggesting that MDSC- targeted inhibition could facilitate the suppression of tumor-specific Treg generation and a resultant increase in the antitumor immune response.

Tregs are a subset of immunosuppressive $\mathrm{T}$ cells that are involved in immune homeostasis and self-tolerance, fetal maternal tolerance, infection, autoimmunity, graftversus-host immunity, and tumor immunity [15]. In the tumor microenvironment, Tregs contribute to the development of immunosuppression, immune evasion and cancer progression [16, 17]. Tregs are recruited to the tumor microenvironment via interactions between tumor-secreted chemokines and their receptors (mainly CCL17/CCL22-CCR4, CCL5-CCR5, CCL28-CCR10 and CXCL9/10/11-CXCR3), converted to a suppressive phenotype by TGF- $\beta$, and efficiently expanded in the presence of tumor-derived IL-10 and TGF- $\beta$ [16]. The accumulation of Tcell-inhibiting [18-20] Tregs in the tumor microenvironment and peripheral blood of cancer patients has been reported in several papers and is correlated with a poor prognosis [21], suggesting that immunotherapeutic approaches targeting Tregs could prolong patient survival.

Although malaria is a serious disease caused by the protozoan genus Plasmodium, several reports have suggested that malaria could stimulate the host innate and adaptive immune systems to combat cancer [22-25] by stimulating IFN- $\gamma$ production, activating natural killer (NK) cells, $\gamma \delta \mathrm{T}$ cells, inducing the maturation of DCs and stimulating T-cell proliferation [24, 26].

We have previously demonstrated that Plasmodium infection significantly suppresses LLC cell growth via the induction of innate and adaptive antitumor responses in a mouse model [22], but it is not yet known whether Plasmodium infection can inhibit the recruitment and activation of MDSCs in the tumor microenvironment. Several studies have been carried out on MDSCs in the peripheral blood of tumor-bearing patients but few studies have focused on tumor-infiltrating MDSCs. The tumor microenvironment is particularly important given that peripheral MDSCs differ from tumor-infiltrating MDSCs in both murine and human cancers [27, 28]. Our current study builds on these findings and further suggests that the induction of innate and adaptive antitumor responses by Plasmodium infection was enhanced, at least in part, through the inhibition of MDSCs and Tregs within the tumor microenvironment.

\section{Materials and methods}

\section{Ethics statement}

The animal experiment facilities were approved by the Guangdong Provincial Department of Science and Technology, and complied with the guidelines of the Animal Care Committee, Guangzhou Institutes of Biomedicine and Health, Chinese Academy of Sciences. All efforts were made to minimize animal suffering.

\section{Sources of animals, cells, and parasites}

Six to eight-week old female C57BL/6 mice were purchased from SLAC Laboratory Animal Company (Shanghai, China) and raised in the animal facility of the Guangzhou Institutes of Biomedicine and Health, CAS. The nonlethal $P$. yoelli 17XNL (Py) strain was a donation from the Malaria Research and Reference Reagent Resource Center (MR4). The murine (LLC) cell line was purchased from ATCC and maintained in RPMI 1640 (Gibco, Carlsbad, CA, USA), supplemented with penicillin $(80 \mathrm{U} / \mathrm{ml})$, streptomycin $(100 \mathrm{U} / \mathrm{ml})$ and $10 \% \mathrm{FBS}$ in a humidified atmosphere of $5 \% \mathrm{CO}_{2}$ at $37^{\circ} \mathrm{C}$.

\section{Animal grouping and inoculation}

For the in vivo experiments, female C57BL/6 mice were randomized into two groups of 5 mice each. To determine the effect of Plasmodium infection on MDSCs and Tregs, we infected tumor-bearing mice (seeded with a subcutaneous (s.c.) injection of $5 \times 10^{5}$ LLC cells), with either Plasmodium yoelli 17XNL parasitized erythrocytes (LP) or an equivalent number of uninfected erythrocytes (LR). Tumors were harvested 18 days post-inoculation for further analysis (Additional file 1). 


\section{Cell preparation from tumor tissues}

Tumor-infiltrating leukocytes were isolated as previously described [29] with modifications. Briefly, tumors were cut into small fragments and digested for $1-3 \mathrm{~h}$ at $37^{\circ} \mathrm{C}$ with 10X Triple enzyme mix. The cells were washed twice and then depleted of red blood cells. The recovered cells were counted, stained and analyzed for myeloid markers using flow cytometry. All antibodies for flow the cytometry analysis were purchased from eBioscience (San Diego, CA, USA). Antibodies were diluted in $\mathrm{PBS} / 1 \% \mathrm{BSA}$, as recommended by the manufacturer, and incubated with the cells for $30 \mathrm{~min}$ at $4{ }^{\circ} \mathrm{C}$.

\section{Sorting of MDSCs and CD8 ${ }^{+}$Tcells from tumor tissues}

Single-cell suspensions were prepared from tumor tissues and stained as previously described. $\mathrm{Ly} 6 \mathrm{G}^{+} \mathrm{Ly} 6 \mathrm{C}^{\text {low }}$ polymorphonuclear cells, Ly6G ${ }^{-}$Ly $6 C^{\text {high }}$ monocytic cells and $\mathrm{CD}^{+} \mathrm{T}$ cells were sorted from tumor tissues isolated from tumor-bearing mice injected with uninfected red blood cells (LR), or parasitized erythrocytes (LP) by fluorescence activated cell sorting (FACS, Beckman MoFlo Astrios).

\section{Flow cytometry}

Cells isolated from tumor tissues were blocked with purified CD16/32 antibody for $10 \mathrm{~min}$ and then stained for MDSCs using anti-CD11b APC, anti-CD45 PE C-Y7, anti-Ly6G PE, and anti-Ly6C FITC antibodies (eBioscience, San Diego, CA) for 30 min on ice. For Tregs staining, the cells were first stained for extracellular Treg markers (CD4 and CD25) with anti-mouse CD4-FITC,and anti-mouse CD25-PE for $30 \mathrm{~min}$. The cells were then fixed using the FOXP3/Transcription factor staining buffer set (eBioscience cat.\# 00-5523) according to the manufacturer's protocol. The cells were subsequently washed and stained with DAPI for $10 \mathrm{~min}$ before analysis by flow cytometry and Flowjo software V10.

\section{RNA extraction and QRT-PCR}

QRT-PCR was performed using tumor tissues and sorted MDSCs and $\mathrm{CD}^{+}$Tcells. Flash-frozen tumor tissues were crushed using a sterile pestle and mortar. Total RNA was then isolated using TRIzol (Invitrogen). Isolated total RNA was reverse transcribed into ${ }_{C}$ DNA using the Prime Script ${ }^{\mathrm{Tm}}$ RT-PCR Kit (Takara). The mRNA expression of VEGF- $\alpha$, GM-CSF, M-CSF, G-CSF, SCF, IL-13, IL-14, CCL-17, CCL-22 and IL-6 in the tumor tissues was analyzed by QRT-PCR. For MDSCs and $\mathrm{CD} 8^{+}$Tcells, the sorted cells were washed with PBS before the isolation of total RNA as described above. The ${ }_{m}$ RNA expression of Arginase 1, NOS2, Survivin, IL-10, and S100A9 in MDSCs was analyzed as described above. The expression levels of granzyme $\mathrm{B}$, perforin, IL-2, IFN-g, PD-1, and TNF- $\alpha$ in $\mathrm{CD}^{+}$Tcells were also analyzed. The relative amount of mRNA in all the analyzed genes was normalized to $\beta$-actin. The primer sequences for the cytokines and other proteins analyzed can be found in Additional file 2: Table S1.

\section{Protein extraction and western blotting}

Total protein was extracted from the isolated MDSCs, exosomes, exosomes-free plasma and $\mathrm{CD} 8^{+}$Tcells using lysis buffer (RIPA) and the protein concentration was determined using the BCA method. A total of $15-20 \mu \mathrm{g}$ of protein was separated by $8-10 \%$ SDS-PAGE and then electrophoretically transferred to a PVDF membrane (Millipore).After the membrane was blocked with $5 \%$ nonfat milk in TBST solution, it was incubated at $4{ }^{\circ} \mathrm{C}$ overnight with primary antibody in blocking solution. The membrane was washed three times with TBST and then incubated at room temperature for $1 \mathrm{~h}$ with horseradish peroxidase-conjugated secondary antibody diluted in TBST. Protein bands were visualized by enhanced chemiluminescence (Pierce) and detected using BioImaging Systems (BIO-RAD ChemiDoc ${ }^{\text {tm }}$ MP Imaging System, USA). Actin protein was used as a loading control. A list of antibodies used for western blotting can be found in Additional file 3: Table S2.

\section{MDSC ROS production assay}

MDSC ROS production was measured using the DCFDA Cellular ROS Detection Assay kit (Abcam, cat \# ab113851) according to the manufacturer's protocol.

\section{MDSC arginase activity}

Arginase activity was measured in cell lysates using the Arginase Activity Assay kit (Abcam, cat.\# ab180877) according to the manufacturer's protocol.

\section{Isolation of exosomes and exosome-free plasma}

Female C57BL/6 mice were randomized into three groups ( $n=20 /$ group). Mice were infected with only Plasmodium yoelli (Py), a combination of LLC and Py (LP), and no infection at all (Naïve). Whole blood was isolated from the respective mice at peak parasitemia (40-45\%), into labelled sterile heparinized tubes and subsequently centrifuged at $3000 \mathrm{~g}$ for $15 \mathrm{~min}$ to remove cells and cell debris. Exosomes were isolated using the Exosomes Isolation kit (SBI System Biosciences cat. \#. EXOQ5TM-1) according to the manufacturer's instructions. Briefly, samples were incubated at room temperature for $30 \mathrm{~min}$, centrifuged at $1500 \mathrm{~g}$ for 30 min, and the exosomes-rich fraction was then washed twice with PBS and either stored at $-80^{\circ} \mathrm{C}$ for future use or directly used in co-culture experiments. The supernatant remaining after exosome isolation from the Py-infected mice was either stored at $-80^{\circ} \mathrm{C}$ for future use or directly used in co-culture experiments. 


\section{Characterization of exosomes}

Isolated exosomes were diluted in PBS (1000 times) and subsequently used for size measurement and analysis on a NanoSight ns300 Malvern (Worcester, UK). Videos of $60 \mathrm{~s}$ for each sample were obtained using the maximum camera gain. Particle size and density were analyzed using the NanoSight particle tracking software (Additional file 4).

\section{In vitro exosomes and red blood cells treatment}

LLC $(3 \times 105)$ cells were plated in triplicate overnight in 6 well plates and incubated under standard conditions to allow for cell attachment. Culture medium was removed and cells were washed twice with PBS. Fresh medium was then added to the cells. $0.2 \mu \mathrm{g} / \mu \mathrm{l}$ of exosomes from different groups and the exosome-free plasma was added to the respective wells and mixed thoroughly. A total of $3 \times 10^{5}$ of either Py-infected RBCs or uninfected RBCs were co-cultured with LLC cells in different wells to determine whether cell-to-cell contact was required for cytokine inhibition. The cells were then incubated for either 24 or $48 \mathrm{~h}$. RNA extraction and QPCR was subsequently performed as earlier described above.

\section{Statistical analysis}

Differences between groups were analyzed for statistical significance using Student's t-test. $P$-valuesless than 0.05 , $0.01,0.001$, and 0.0001 were considered statistically significant, and indicated by ${ }^{*},{ }^{* *},{ }^{* * * *}$ and ${ }^{* * * * *}$ respectively, in each figure. Representative results from three independent experiments with similar results are shown.
Statistical analysis was performed using GraphPad Prism (version 6).

\section{Results \\ Plasmodium infection significantly reduced the proportion of MDSCs in the tumor microenvironment}

To examine the MDSC subsets in the tumor microenvironment, single cell suspensions were prepared from the tumor tissues of Plasmodium. yoelli 17XNL (Py)-treated and untreated tumor-bearing mice, stained for MDSC markers and analyzed by flow cytometry. After Live/ Dead,- and $\mathrm{CD} 45^{+} \mathrm{CD}_{11 b^{+}}$gating (Fig.1a), Ly6G ${ }^{+} \mathrm{Ly} 6 \mathrm{C}^{\text {low }}$ polymorphonuclear and $\mathrm{Ly}^{6} \mathrm{G}^{-} \mathrm{Ly} 6 \mathrm{C}^{\text {high }}$ monocytic cells were gated (Fig.1b\&c) and analyzed( $n=5$ /group). Our data indicated a significant reduction in the percentage of MDSCs (Fig.1d) $(P<0.01)$ in the tumor tissues of tumor-bearing mice treated with Py-infected RBCs compared to the control.

Several reports have shown that MDSCs are not evenly distributed in the tumor microenvironment in terms of number or immunosuppressive capability. We wanted to determine the relative proportions of the two major MDSC subsets (P-MDSCs and M-MDSCs) in the tumor microenvironment irrespective of Plasmodium treatment. Our flow cytometry analysis indicated a higher proportion of P-MDSCs than M-MDSCs in the tumor tissues of both the untreated and treated mice (Fig.1e). More importantly, there was a more pronounced reduction in the M-MDSCs than the P-MDSCs upon treatment. Because M-MDSCs are believed to play a more prominent immunosuppressive role in the tumor microenvironment than P-MDSCs, it is

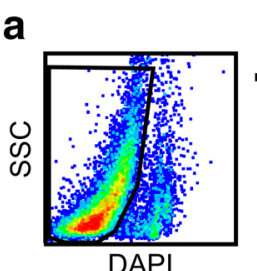

d

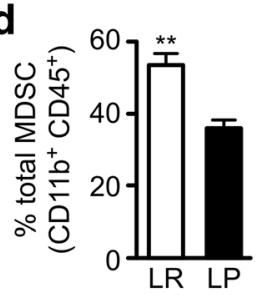

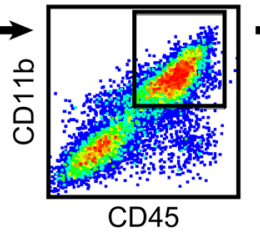

e

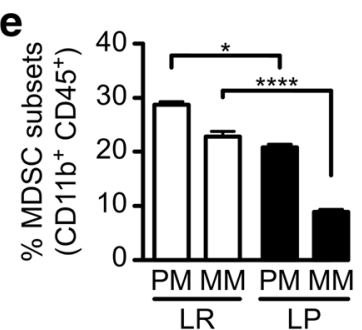

b $\quad L R$

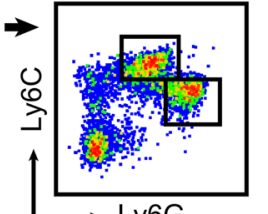

C

LP

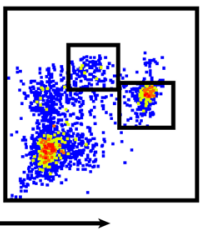

f

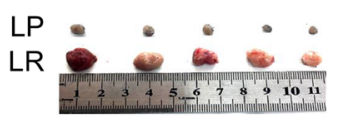

Fig. 1 Downregulation of the population of MDSCs in the tumor microenvironment by Plasmodium. a Gating protocol for the identification of MDSC subsets. b Gated polymorphonuclear and monocytic MDSCs in the tumor tissues of uninfected tumor-bearing mice (LR, control). c Gated polymorphonuclear and monocytic MDSCs in the tumor tissues of Py-infected tumor-bearing mice (treated, LP). $\mathbf{d}$ Comparison of the total MDSC population in the tumor tissues of both the treated and the control groups. e The relative proportions of polymorphonuclear and monocytic MDSC subsets in the tumor tissues of tumor-bearing mice treated with infected and uninfected RBCs. $\mathbf{f}$ Tumors harvested from tumor-bearing mice treated or untreated with Plasmodium 18 days after tumor inoculation. A total of 50,000 live events was recorded and analyzed per sample. The average number of MDSCs gated out were 2000 cells and 5000 cells for (LP) and (LR) respectively 
expected that Plasmodium infection might substantially inhibit MDSC-mediated immunosuppression in the tumor microenvironment. Careful observation of the isolated tumor tissues 18 days after tumor inoculation showed a remarkable reduction in the tumor size of Py-treated mice compared to that of the control mice (Fig. 1f).

Plasmodium infection significantly reduced the proportion of Tregs in the tumor microenvironment

Our previous work [22] revealed that Plasmodium infection significantly upregulated the population of $\mathrm{CD} 4+$, $\mathrm{CD} 8+$, and granzyme-producing CD8+ Teff cells in the peripheral blood, tumor-draining lymph nodes and tumor tissues in mice. However, it is yet to be understood if Plasmodium infection could inhibit immunosuppressive Tregs in the tumor microenvironment. The accumulation of Tregs have been shown to correlate with diminished survival in cancer patients. We therefore investigated whether Plasmodium infection could inhibit the accumulation of Tregs in the tumor microenvironment. Single-cell suspensions were prepared from tumor tissues as previously described for MDSCs and subsequently stained for Treg markers. After gating out the $\mathrm{DAPI}^{+}$ $\mathrm{CD}^{+}$subset (Fig. 2a), the population of $\mathrm{CD} 25^{+} \mathrm{FOXP3}^{+-}$ cells (Fig. 2b \& c) was gated. Our results indicated a significant reduction in the population of CD25 + FOXP3 + cells in the tumor tissues of Py-treated mice compared to those of the control mice (Fig. $2 \mathrm{~d})(P<0.001)$.

Infection with Plasmodium inhibits the expression of key MDSC- and Treg-recruiting cytokines, chemokines and growth factors in the tumor microenvironment

MDSCs are recruited to the tumor microenvironment via tumor-secreted cytokines, chemokines and growth factors. To test the effects of Plasmodium infection on the secretion of tumor-derived cytokines and chemokines in the tumor microenvironment, we carried out QRT-PCR on tumor samples isolated from tumor-bearing mice treated with either parasitized or unparasitized erythrocytes. Our results showed a significant reduction in the relative mRNA expression of GMCSF (Fig. 3a) $(P<0.01)$, GCSF(Fig. 3b) $(P$ $<0.01)$, MCSF(Fig. 3c) $(P<0.001)$, IL-1 $\beta$ (Fig. 3d) $(P<0.01)$, VEGF(Fig. 3e) $(P<0.05)$, IL-6(Fig. 3f) $(P<0.05)$, IFN-g (Fig. 3g) $(P<0.01)$, IL-14(Fig.3h) $(P<0.01)$, IL-13(Fig. 3i) $(P<$ $0.01)$, TGF- $\beta$ (Fig. 3j) $(P<0.01)$, CCL-17(Fig. 3k) $(P<0.01)$ and CCL-22(Fig. 3l) $(P<0.01)$ in the Py-treated group compared to the control group suggesting that Plasmodium infection could inhibit the generation of immunosuppressive cells in the tumor microenvironment. Furthermore, western blot analysis revealed a reduction in the expression of the CCR4 (Fig.3m) receptor in the tumors of Py-treated mice compared to those of the untreated mice. Several reports have implicated the CCL17/22-CCR4 pathway and tumor-derived TGF- $\beta$ in Treg recruitment and activation respectively in the tumor microenvironment [30, 31], suggesting that inhibition of the CCL17/22-CCR4 pathway could substantially modulate the tumor accumulation of Tregs.

MDSCs isolated from the tumors of Plasmodium-infected mice showed inhibited phosphorylation of STAT proteins Available evidence indicates that the STAT3 pathway is crucial for MDSC differentiation, survival and immunosuppressive functions [32]. Several reports have indicated that activated STAT3 modulates the expression of several target genes involved in cell cycle regulation, angiogenesis, tumor invasiveness and apoptosis [33-35], and that the disruption of STAT3 in myeloid cells alters a

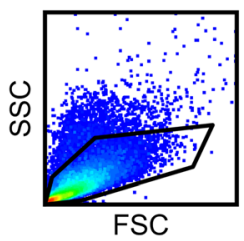

d

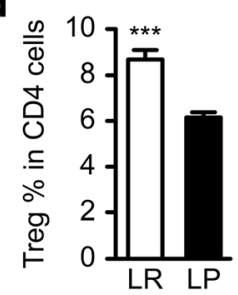

b
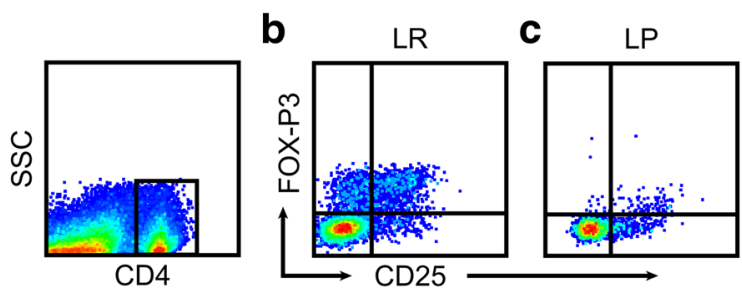

Fig. 2 Effects of Plasmodium on the population of Tregs in the tumor microenvironment. a Gating protocol for CD4 ${ }^{+}$cells. b Gated CD25 ${ }^{+} \mathrm{FOXP3}^{+}$Treg population in untreated tumor tissues. c Gated CD25 ${ }^{+} \mathrm{FOXP3}{ }^{+}$Treg population in treated tumor tissues. $\mathbf{d}$ The populations of Tregs in the tumors were compared. Plasmodium infection significantly downregulated the populations of Tregs in the tumor tissues of tumorbearing mice compared to the control mice. ${ }^{* *} P<0.001$ 


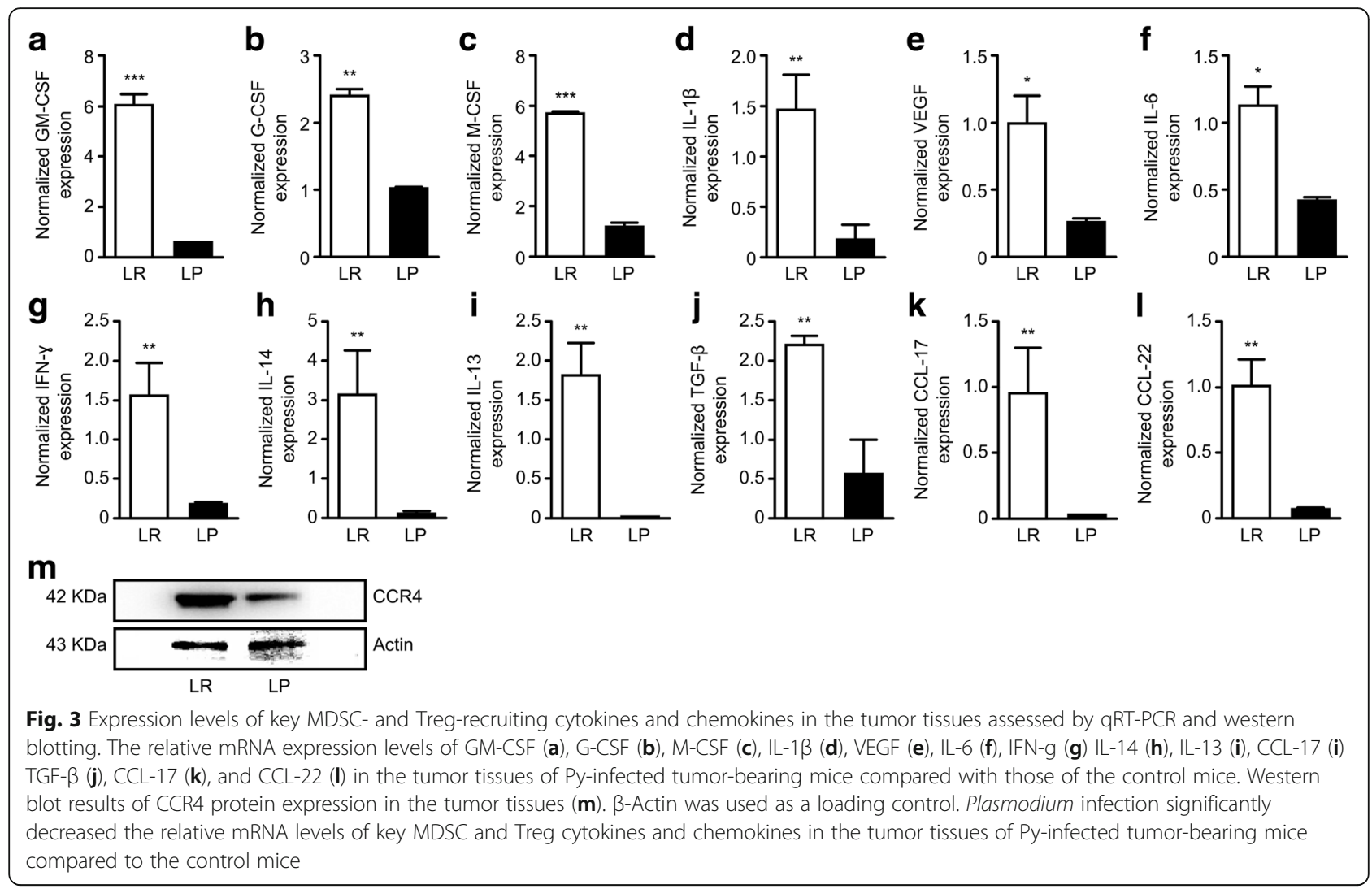

immunosuppressive cell abundance [36]. We therefore examined whether Plasmodium infection could inhibit the phosphorylation of STAT3 and other STAT pathways including those of their downstream proteins - especially those involved in cell survival and immunosuppression. We used western blotting to assess the protein expression levels of p-STAT1, pSTAT3, pSTAT5, pSTAT6, NF-kB, Survivin and S100A9 and QRT-PCR to assess Survivin and S100A9. Our western blot results showed that, compared to the control, Plasmodium treatment inhibited the protein expression of p-STAT1, pSTAT3, pSTAT5, pSTAT6, NF-kB, Survivin and S100A9 (Fig.4a). Because the subsets of MDSCs have different immunosuppressive potentials in the tumor microenvironment and because STAT3 is the most crucial factor for MDSC activation, we sorted the individual subsets (P-MDSCs and M-MDSCs) for additional western blot analysis. Our results indicated that Plasmodium treatment inhibited the expression of pSTAT3 protein in each of the MDSC subsets analyzed (Fig.4d). Furthermore, we carried out QRT-PCR on the sorted MDSCs to examine the mRNA expression of two downstream proteins (Survivin and S100A9). Our results indicated that Plasmodium treatment downregulated the mRNA expression level of Survivin (Fig.4b) $(P<0.05)$, and S100A9 (Fig.4c) $(P<0.05)$ compared to the control. These results suggest that Plasmodium infection could modulate MDSC proliferation and survival through inhibition of the STAT pathways.
MDSCs from the tumors of Plasmodium-infected mice have significantly reduced expression of immunosuppressive molecules

MDSCs are known to inhibit Tcells through the secretion of arginase 1, iNOS, and ROS in the tumor microenvironment $[4,37]$. Thus, we next investigated whether Plasmodium infection affected the ability of MDSCs to express or secrete T-cell-inhibiting molecules in the tumor microenvironment. Ly6G ${ }^{+}$Ly $6 C^{\text {low }}$ granulocytic and Ly6G ${ }^{-}$Ly6 $C^{\text {high }}$ monocytic cells were isolated from the tumor tissues of tumor-bearing mice treated with either parasitized or unparasitized erythrocytes and used for the analysis of immunosuppressive molecules by QRT-PCR and functional assays. Our QRT-PCR results indicated that the mRNA expression levels of IL-10(Fig.5a) $(P<0.01)$, iNOS (Fig.5b) $(P<0.0001)$ and, Arginase 1(Fig.5c) $(p<0.01)$ were downregulated in the treatment group compared to the control group. We further tested the functional status of ROS and Arginase 1 in the isolated MDSCs. We found a significant reduction in ROS activity (Fig.5d) $(P<0.01)$ and Arginase activity (Fig.6e) $(P<0.01)$ in the treated group compared to the control group. These results indicate that Plasmodium infection could modulate the immunosuppressive capabilities of MDSCs in the tumor microenvironment by inhibiting the secretion of immunosuppressive molecules. 


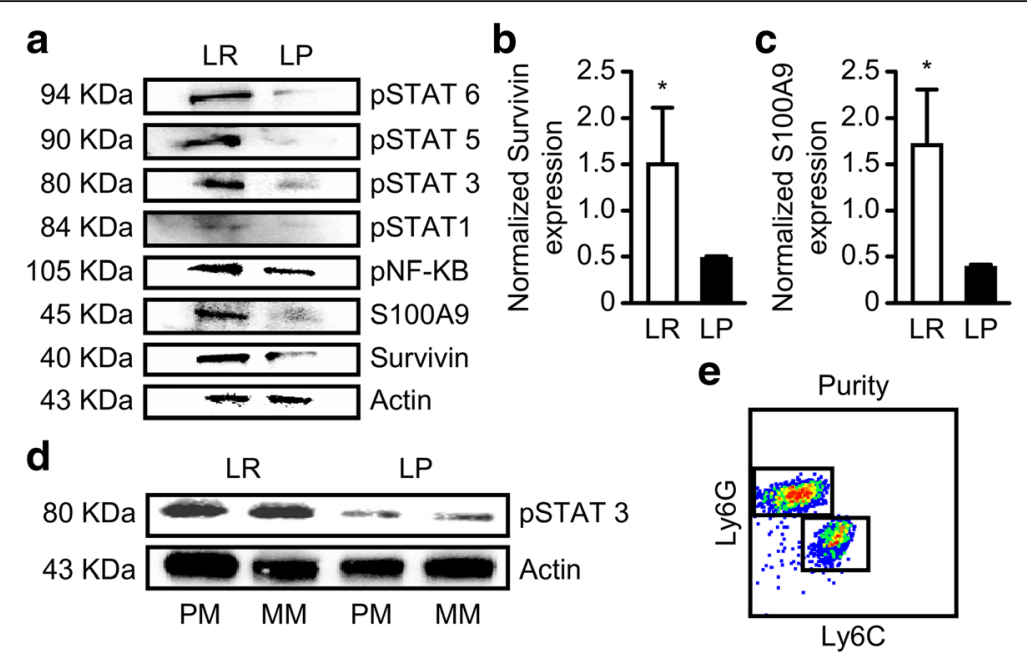

Fig. 4 Effects of Plasmodium infection on MDSC signal transduction and downstream protein expression. The expression levels of pSTAT1, pSTAT3, pSTAT5, pSTAT6, NF-KB, Survivin, and S100A9 were assessed by qRT-PCR and western blot analysis. a The protein expression levels of pSTAT1, pSTAT3, PSTAT5, pSTAT6, NF-KB, Survivin and S100A9 in MDSCs from the infected and control mice, as determined by western blotting. b, $\mathbf{c}$ qRT-PCR analysis of Survivin and S100A9 expression of the sorted MDSCs. $\mathbf{d}$ The protein expression of pSTAT3 in the two MDSC subsets was assessed. (e) Purity of the sorted MDSCs. $\beta$-Actin was used as a loading control for both the qRT-PCR and western blot analysis. ${ }^{*} P<0.05$. MM, monocytic MDSC; PM, polymorphonuclear MDSC

\section{$\mathrm{CD}^{+} \mathrm{T}$ cells in the tumor tissues of Plasmodium-treated} mice expressed high levels of cytotoxic molecules and low levels of PD-1

$\mathrm{CD}^{+}$Tcells effect tumor killing in two ways; (i) through the secretion of cytotoxic molecules (granzyme B and perforin) and, (ii) through the secretion of proinflammatory cytokines (IL-2, TNF- $\alpha$, and IFN-g). We therefore examined whether the inhibition of MDSCs and Tregs in the tumor microenvironment could promote the tumor cell-killing ability of $\mathrm{CD}^{+}$Tcells. We isolated $\mathrm{CD}^{+} \mathrm{T}$ cells from the tumors and carried out QRT-PCR. Interestingly, we found that the $\mathrm{CD}^{+}$Tcells from the tumors of Plasmodium-treated mice expressed remarkably higher levels of perforin and granzyme B (Fig. 6b
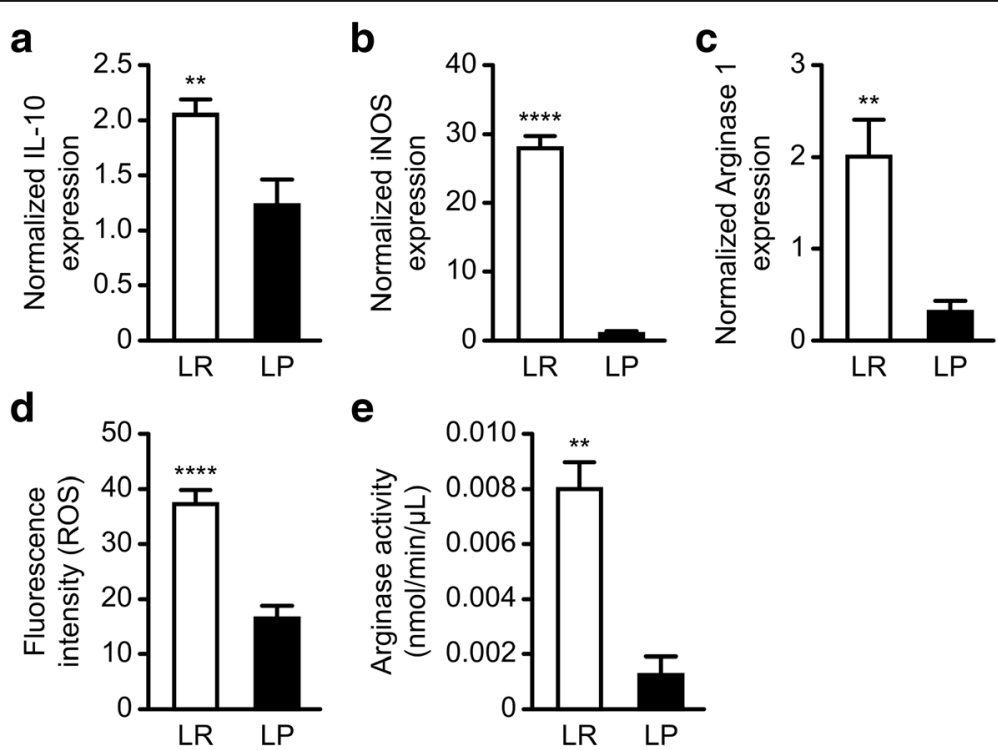

Fig. 5 Effects of Plasmodium infection on the expression of immunosuppressive molecules by sorted MDSCs. The MDSC expression of IL-10, arginase 1, NOS2, and ROS was assessed by qRT-PCR and functional assays. Relative mRNA expression levels of IL-10 (a), NOS2 (b), and arginase 1 (c) of MDSCs isolated from the tumor tissues of Py-treated and untreated tumor-bearing mice. The levels of ROS (d) and arginase activity (e) were detected using a DCFDA Cellular ROS Detection Assay Kit (Abcam; cat. \# ab113851) and Arginase Activity Assay Kit (Abcam; cat. \# ab180877), respectively. ${ }^{* *} P<0.01,{ }^{* * *} P<0.0001$ 
a

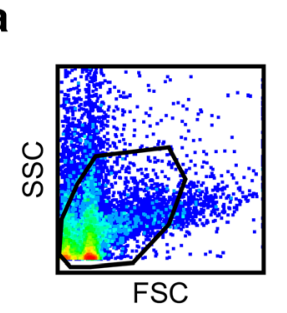

b

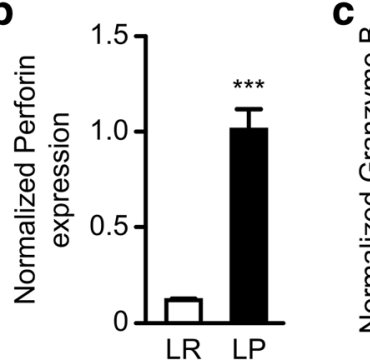

e

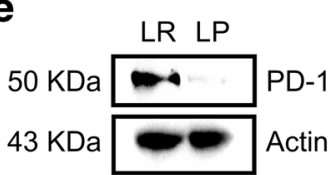

d

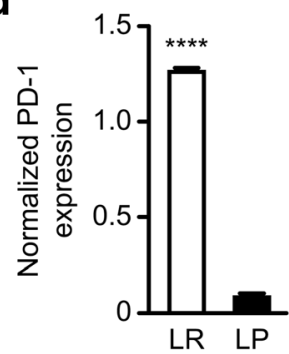

Fig. 6 Effects of Plasmodium infection on $C D 8^{+} T$ cells in the tumor microenvironment. a Flow cytometry gating protocol for $C D 8^{+} T$ cell sorting. b Comparison of the mRNA expression of PD-1 on sorted CD8 ${ }^{+} T$ cells. $\mathbf{c}$ mRNA expression of granzyme $\mathrm{B}(\mathbf{d})$ and perforin by the sorted $\mathrm{CD} 8^{+} \mathrm{T}$ cells. e Protein expression of PD-1 on sorted $C D 8^{+} T$ cells determined by western blotting. ${ }^{* *} \mathrm{P}<0.01$

\&c). However, we observed that Plasmodium infection did not promote the ability of $\mathrm{CD} 8^{+} \mathrm{T}$ cells to produce proinflammatory cytokines (IL-2, IFN-g, and TNF- $\alpha$; data not shown). Because the B7-H1(PD-L1)/PD-1 signaling pathway is believed to play a key role in $\mathrm{T}$ cell exhaustion [38], we analyzed the PD-1 expression of sorted $\mathrm{CD}^{+}$Tcells by QRT-PCR and western blotting. We found a remarkable reduction in the expression of PD-1 on $\mathrm{CD}^{+}$Tcells in the tumor tissues at both the mRNA (Fig. 6d) and protein (Fig. 6e) levels. These results suggest that Plasmodium infection could promote $\mathrm{CD} 8^{+} \mathrm{T}$ cell-mediated tumor cell-death in the tumor microenvironment through the upregulation of granzyme B and perforin while preventing inhibition through the PD-1/ PD-L1 signaling pathway.

\section{Plasmodium infection inhibits tumor cytokines and chemokines secretions through exosomes-like vesicles} Plasmodium sporozoites injected into the blood stream have demonstrated high affinity for liver cells due to the availability of highly sulfated heparan sulphate proteoglycans (HSPG) [39]. This therefore suggests that interactions between Plasmodium and the tumor could be mediated via the infected red blood cells or through the release of microvesicles. We asked if the inhibition of tumor cytokine and chemokine secretion is mediated by cell-to-cell contact or through the release of microvesicles. Our results indicated that Plasmodium infection significantly inhibits the tumor secretions of GMCSF, IL-10, IL-6, CCL-17, and CCL-22 in vitro (Fig. 7a-f) through exosomes-like vesicles released by infected red blood cells.

\section{Discussion}

Tumor growth is often associated with both the stimulation of antitumor $\mathrm{T}$ cell responses and a parallel induction of immune suppression. The balance of these two events determines, to a large extent, the prognosis of the disease. In cancer patients, the tumor-specific Tcell response has been found to correlate with improved outcomes [40-44]. However, the tumor-specific immune response is compromised by the recruitment of immunosuppressive cellular and molecular mechanisms within the tumor microenvironment. Several reports have indicated that immunosuppressive cells mobilized into the tumor microenvironment by tumor-derived factors and cytokines are a major cause of the failure of novel immunotherapies and chemotherapies [45-47]. Among these cellular infiltrates, MDSCs and Tregs are believed to play a major role in the suppression of antitumor immune responses [48-50]. Srivastava et.al, reported that targeting MDSCs could augment the antitumor immune response against lung cancer [51]. We previously reported that Plasmodium infection could induce potent innate and adaptive antitumor immune responses in a lung cancer mouse model [22], but the effect of Plasmodium infection on the immunosuppressive abilities of MDSCs and Tregs in the tumor microenvironment is not yet known.

In the current study, we provide evidence that Plasmodium infection could inhibit the recruitment and activation of MDSCs and Tregs in the tumor microenvironment in a variety of ways, including the inhibition of STAT phosphorylation (Additional file 5). We demonstrated that Plasmodium infection downregulated the secretion of several MDSC- and Treg- recruiting cytokines and growth 


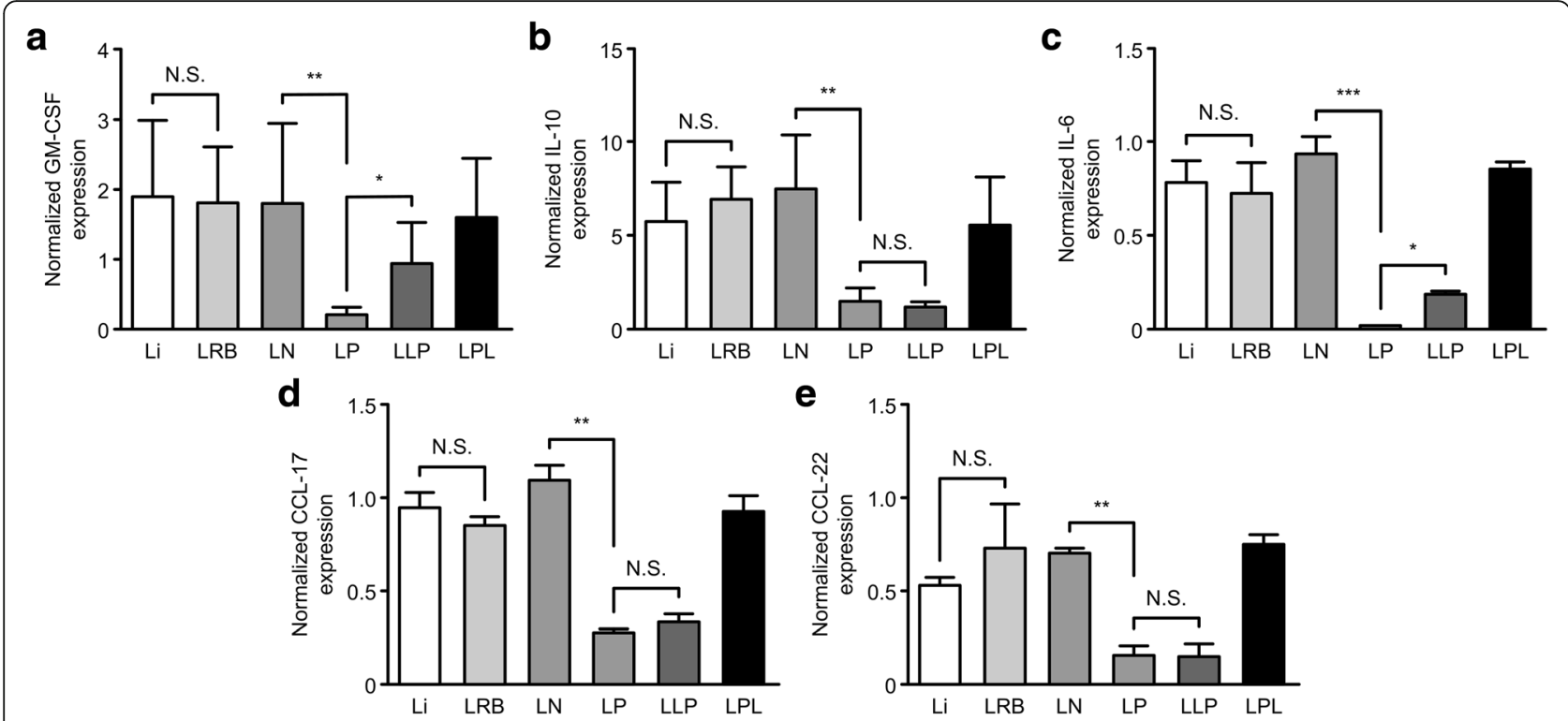

Fig. 7 Plasmodium infection inhibits tumor cytokines and chemokines secretions through exosomes-like vesicles. To determine whether or not cell-to-cell contact was required for the inhibition of tumor cytokines, we co-cultured LLC cells with either infected RBCs (Li), uninfected RBCs $(L R B)$, exosomes isolated from uninfected mice $(L N)$, exosomes isolated from Py-infected mice (LP), exosomes isolated from tumor-bearing mice infected with Py (LLP), or plasma from Py-infected mice after exosomes have been isolated (LPL). Cells were cultured for either 24 or $48 \mathrm{~h}$ and subsequently harvested for RNA extraction and QPCR analysis. Our QPCR results suggest that infected RBCs does not communicate with tumor cells through cell-to-cell contact. Exosome-like vesicles significantly inhibited tumor release of GMCSF(a), IL-10(b), IL-6(c), CCL-17(d), and CCL-22(e) at the mRNA levels

factors in the tumor microenvironment which is consistent with the reduction in the proportion of MDSCs and Tregs in the tumor tissues of the Py-treated tumor-bearing mice (Figs. 1 \& 2). The significant reduction in the proportion of MDSCs and Tregs in the tumor tissues of the treatment group may partially explain the remarkable reduction in tumor size observed (Fig. 1f), potentially due to enhanced T-cell-mediated tumor cell-death orchestrated by the inhibition of MDSC- and Tregs-mediated immune suppression. This finding corroborates other findings demonstrating the efficacy of strategies targeting MDSCs and Tregs [52, 53].

We further observed that P-MDSCs out-numbered M-MDSCs in the tumor tissues of both the treated and untreated tumor-bearing mice (Fig.1e). Although M-MDSCs are believed to play a dominant role in the tumor microenvironment, the relatively higher proportion of P-MDSCs may be due to the ability of M-MDSCs to rapidly differentiate into tumor-associated macrophages(TAMs, reviewed in [12]).

A major pathway in the recruitment of Tregs into the tumor microenvironment is the CCL17/22-CCR4 pathway. We observed that Plasmodium infection significantly downregulated the expression of the chemokines CCL17 and CCL22 as well as their receptor, CCR4 in the tumor microenvironment (Fig. 3k-m) further validating the reduction in the proportion of Tregs in the tumor tissues.
Available evidence points to the fact that MDSCs and Tregs contribute significantly to immune regulation in the tumor microenvironment and impair the long-term efficacy of current combination therapies for lung cancers [54]. The activation of STAT signaling in myeloid cells promotes cell proliferation and prevents apoptosis through the expression of Survivin, S100A9, Bcl-xL, c-myc, or cyclin D1 [55]. In our study, MDSCs isolated from the tumors of Py-treated mice showed significant reduction in the expression of phosphorylated STAT proteins, S100A9, and Survivin at both the mRNA and protein levels, further explaining their reduced proportion in the tumor tissues.

One of the ways MDSCs contribute to immunosuppression in the tumor microenvironment is by generating ROS, Arginase 1, free radicals and cytokines to suppress host CD4+ and CD8+ Tcell responses [8, 56, 57].It is believed that Arginase and iNOS function synergistically in MDSCs to inhibit antigen-specific T cell response $[58,59]$. It has been demonstrated that the production of ROS by MDSCs could downregulate the $\zeta$-chain on Tcells and induce Tcell tolerance $[46,60]$. The ability of Plasmodium infection to perturb ROS generation, inhibit arginase activity, and downregulate iNOS and IL-10 expression clearly demonstrates the potential benefits of the Plasmodium-mediated targeting of immunosuppressive cells in the tumor microenvironment. 
$\mathrm{CD}^{+} \mathrm{T}$ cell-mediated immunity plays a vital role in limiting cancer initiation and progression. At steady state, $\mathrm{CD} 8^{+} \mathrm{T}$ cells can eliminate cancer cells by releasing cytotoxic granules containing granzyme and perforin and at the same time promote inflammation by releasing proinflammatory cytokines [61]. IFN $\gamma$ released by $\mathrm{CD}^{+} \mathrm{T}$ cells promotes the tumor expression of MHC class 1 antigens thereby making tumors susceptible to immune cell detection and elimination [62]. Interestingly, our results indicated that sorted $\mathrm{CD}^{+}$cytotoxic Tcells expressed remarkably high levels of granzyme $\mathrm{B}$ and perforin indicating a high tumor cell-killing potential. However, there were no observable increases in the expression of the proinflammatory cytokines tested (IL-2, TNF- $\alpha$, and IFNY) within the tumor tissues. Although it was impossible for us to measure the cytokine levels in the tumor microenvironment at the early days of infection(1-7 days post infection), our current results may be a reflection of our earlier report that Plasmodium infection upregulates the production of proinflammatory cytokines(IL-2, IFN $\gamma$ and TNF- $\alpha$ ) in peripheral blood during the early stage of infection, peaks at day 7 , and declines to a minimal level 14 days post infection [63]. Because the tumor samples were harvested 18 days after Plasmodium injection, this timing may account for the reduced expression of proinflammatory cytokines by $\mathrm{CD}^{+} \mathrm{T}$ cells in the tumor microenvironment.

It is believed that intratumoral $\mathrm{T}$ cells demonstrate exhaustion by expressing high levels of inhibitory surface molecules such as PD-1, CD160, CTLA-4, LAG-3, TIM-3 and BTLA that can prevent T cell activation. Among those inhibitory molecules, researchers believe that those of the PD-1/PD-L1 signaling pathway plays a key role in $\mathrm{CD} 8^{+}$Tcell functional exhaustion. We therefore tested the expression of PD-1 in the sorted CD8 ${ }^{+}$Tcells by QRT-PCR and western blotting. Interestingly, our results indicated a significant reduction in the expression of PD-1 on $\mathrm{CD} 8^{+}$ T cells (Fig. 6d), highlighting the potential of utilizing Plasmodium infection to develop effective immunotherapies.

The asexual phase of the Plasmodium life cycle is initiated by the injection of sporozoites into the mammalian host. Sporozoites are known to rapidly invade the liver, develop in the liver and are released as merozoites that invades red blood cells within minutes [64]. Therefore, interactions between Plasmodium and tumor cells can only be mediated through the infected red blood cells or through microvesicles secreted from infected red blood cells. Our co-culture experiments revealed that infected and uninfected red blood cells did not inhibit tumor cytokine and chemokines secretion, suggesting that cell-to-cell contact was not involved in this inhibition. In contrast, exosomes from infected red blood cells significantly inhibited the secretion of cytokines and chemokines from the tumor cells at the messenger RNA levels when compared to the controls. The release of exosome-like vesicles from cells under stress conditions have since been reported in several papers. Interestingly, Plasmodium falciparum-infected RBCs have been demonstrated to directly communicate through exosome-like vesicles capable of delivering genes [65].

Further work should focus on the specific proteins, molecules, microRNAs and/or long noncoding RNAs in the exosomes responsible for these Plasmodium-me diated effects with the aim of developing effective immunotherapeutic strategies against lung cancer.

\section{Conclusions}

In conclusion, we report that Plasmodium infection inhibits the accumulation of immunosuppressive MDSCs and Tregs in the tumor microenvironment in a murine lung cancer model. The inhibition of MDSC STAT phosphorylation, the downregulation of immunosuppressive molecules, the elevated expression of granzyme $\mathrm{B}$ and perforin by $\mathrm{CD}^{+}$Tcells and the inhibition of PD- 1 expression on $\mathrm{CD} 8^{+}$Tcells clearly demonstrate the relevance of these findings and their merit for further investigation. We further demonstrated that Plasmodium infection inhibits tumor secretion of cytokines and chemokines through the release of exosome-like vesicles rather than through cell-to-cell contact. This study advances our understanding of the effect of Plasmodium infection in enhancing the host immune response to lung cancer and provides insight into the development of potent immunotherapeutic strategies. Subsequent studies will focus on the identification of specific molecules in the exosomes that are responsible for the inhibition of the cytokine signaling in tumor cells.

\section{Additional files}

Additional file 1: Figure S2. Schematic diagram summarizing the experimental design. (TIF $268 \mathrm{~kb}$ )

Additional file 2: Table S1. List of primers used for qRT-PCR. (DOCX $161 \mathrm{~kb}$ ) Additional file 3: Table S2. List of antibodies used for western blotting and the companies from which they were obtained. (DOCX $12 \mathrm{~kb}$ )

Additional file 4: Figure S3. Characterization of exosomes by Nanosight. (a)Analysis of the sizes of exosomes isolated from the plasma of mice infected with Py (Py ex) and uninfected mice ( $\left.\mathrm{N} \mathrm{ex}){ }^{* *} P<0.01\right)$. (b) Analysis of the particles number of exosomes isolated from the plasma of mice infected with Py (Py ex) and uninfected mice ( $\mathrm{N}$ ex). $\left({ }^{*} P<0.05\right)$. (C) Representative graph obtained from Nanosight data. (TIF $337 \mathrm{~kb}$ )

Additional file 5: Figure S1. Graphical abstract of Plasmodium modulation of MDSC- and Treg-mediated regulation of $\mathrm{CD}^{+} \mathrm{T}$ cells in the tumor microenvironment. Tumor cytokines and chemokines inhibit the differentiation of myeloid cells in the tumor microenvironment, leading to the accumulation of MDSCs. The binding of cytokines to their receptors on MDSCs triggers the phosphorylation of several signal transduction molecules and the activation of transcription, resulting in the expression of downstream proteins. MDSCs express arginase 1, ROS, and iNOS, which inhibit cytotoxic T lymphocytes. The expression of anti-apoptosis proteins, Survivin and S100A9 enables MDSC proliferation and accumulation. Tumor-secreted cytokines convert naïve $\mathrm{CD}^{+} \mathrm{T}$ cells to Tregs in the tumor microenvironment. Tregs further inhibit CTLs by 
releasing molecules such as IL-10 and TGF- $\beta$. (G, cytokines; GR, cytokine receptors). Plasmodium infection inhibits tumor-derived cytokine and chemokine secretion in the tumor microenvironment, thereby inhibiting the conversion of myeloid cells to MDSCs, the expression of downstream proteins, the conversion of naive $\mathrm{CD4}^{+} \mathrm{T}$ cells to Tregs, and the expression of PD-1 on cytotoxic T cells. (TIF $1593 \mathrm{~kb}$ )

\section{Abbreviations}

DC: dendritic cells; iNOS: inducible nitric oxide synthase; LLC: Lewis lung cancer cells; MDSCs: myeloid derived suppressive cells; M-MDSC: monocytic myeloid derived suppressor cells; MØ: macrophages; NK: natural killer cells; PD-1: programmed cell death 1; PD-L1: programmed cell death ligand 1; PMDSC: polymorphonuclear myeloid derived suppressive cells; Py: plasmodium yoelli; GPCR/QRT-PCR: quantitative PCR; ROS: reactive oxygen species; STAT: signal transducer and activator of transcription; TAM: tumorassociated macrophages; Tregs: regulatory T cells

\section{Acknowledgments}

D.A is sponsored by the UCAS Fellowship for international PhD students. We want to thank Dr. Liu Guanjie, and Mr. Zhong Kwei for their assistance in the flow cytometry analysis.

\section{Funding}

This study was supported by the National Natural Science Foundation of China (Nos.31570925 and 81673003), the Science and Technology Project of Guangzhou City (No.201707010447), and the Ministry of Science and Technology Key Program (No. 2016YFE0107300).

\section{Availability of data and materials}

All data generated or analyzed during this study are included in this published article and its Additional files.

\section{Authors' contributions}

D.A. conceived, planned and carried out this work. Y.Y. L.Q., Z.T. and K.G. helped in the animal experiments. L.D., S. Z and X.L. verified the analytical methods and performed the computations. L.O. and X.C. supervised the findings of this work. All authors discussed the results and contributed to the final manuscript. All authors read and approved the final manuscript.

\section{Ethics approval}

The animal experiment facilities were approved by the Guangdong Provincial Department of Science and Technology, and complied with the guidelines of the Animal Care Committee, Guangzhou Institutes of Biomedicine and Health, Chinese Academy of Sciences. All efforts were made to minimize animal suffering.

\section{Consent for publication}

Not applicable.

\section{Competing interests}

The authors declare that they have no competing interests.

\section{Publisher's Note}

Springer Nature remains neutral with regard to jurisdictional claims in published maps and institutional affiliations.

\section{Author details}

State Key Laboratory of Respiratory Disease, Center of Infection and Immunity, Guangzhou Institutes of Biomedicine and Health, Chinese Academy of Sciences, 190 Kaiyuan Avenue, Science Park, Guangzhou 510530, People's Republic of China. ${ }^{2}$ University of Chinese Academy of Sciences, No. 190 Yuquan Road, Beijing 100049, People's Republic of China. ${ }^{3}$ Guangzhou Regenerative Medicine and Health-Guangdong Laboratory GRMH-GDL, Guangzhou 510530, People's Republic of China.

Received: 18 February 2019 Accepted: 14 March 2019 Published online: 12 April 2019

\section{References}

1. Coussens LM, Werb Z. Inflammation and cancer. Nature. 2002;420:860-7.
2. Gattinoni L, Powell DJ Jr, Rosenberg SA, Restifo NP. Adoptive immunotherapy for cancer: building on success. Nat Rev Immunol. 2006;6:383-93.

3. Li Q, Pan PY, Gu P, Xu D, Chen SH. Role of immature myeloid gr-1+ cells in the development of antitumor immunity. Cancer Res. 2004;64:1130-9.

4. Rodriguez PC, et al. Arginase I in myeloid suppressor cells is induced by COX-2 in lung carcinoma. J Exp Med. 2005;202:931-9.

5. Serafini $\mathrm{P}$, et al. Phosphodiesterase-5 inhibition augments endogenous antitumor immunity by reducing myeloid-derived suppressor cell function. Exp Med. 2006;203:2691-702.

6. Curiel TJ. Tregs and rethinking cancer immunotherapy. J Clin Invest. 2007; 117:1167-74.

7. Kusmartsev SA, Li Y, Chen SH. Gr-1+ myeloid cells derived from tumorbearing mice inhibit primary $T$ cell activation induced through $C D 3 / C D 28$ costimulation. J Immunol. 2000;165:779-85.

8. Gabrilovich DI, Nagaraj S. Myeloid-derived suppressor cells as regulators of the immune system. Nat Rev Immunol. 2009:9:162-74.

9. Talmadge JE, Gabrilovich DI. History of myeloid-derived suppressor cells. Nat Rev Cancer. 2013:13:739-52.

10. Marvel D, Gabrilovich DI. Myeloid-derived suppressor cells in the tumor microenvironment: expect the unexpected. J Clin Invest. 2015;125:3356-64.

11. Ugel S, De Sanctis F, Mandruzzato S, Bronte V. Tumor-induced myeloid deviation: when myeloid-derived suppressor cells meet tumor-associated macrophages. J Clin Invest. 2015;125:3365-76.

12. Kumar V, Patel S, Tcyganov E, Gabrilovich DI. The nature of myeloidderived suppressor cells in the tumor microenvironment. Trends Immunol. 2016;37:208-20.

13. Djeu J, Wei S. Chemoimmunomodulation of MDSCs as a novel strategy for cancer therapy. Oncoimmunology. 2012;1:121-2.

14. Pan PY, et al. Immune stimulatory receptor CD40 is required for T-cell suppression and T regulatory cell activation mediated by myeloid-derived suppressor cells in cancer. Cancer Res. 2010;70:99-108.

15. Sakaguchi S, Yamaguchi T, Nomura T, Ono M. Regulatory T cells and immune tolerance. Cell. 2008:133:775-87.

16. Nishikawa H, Sakaguchi S. Regulatory T cells in cancer immunotherapy. Curr Opin Immunol. 2014:27:1-7.

17. Elkord $\mathrm{E}$, et al. T regulatory cells in cancer: recent advances and therapeutic potential. Expert Opin Biol Ther. 2010;10:1573-86.

18. Kakita $\mathrm{N}$, et al. Comparative analyses of regulatory $\mathrm{T}$ cell subsets in patients with hepatocellular carcinoma: a crucial role of CD25(-) FOXP3(-) T cells. Int J Cancer. 2012;131:2573-83.

19. Amedei $A$, et al. Ex vivo analysis of pancreatic cancer-infiltrating $T$ lymphocytes reveals that ENO-specific Tregs accumulate in tumor tissue and inhibit Th1/Th17 effector cell functions. Cancer Immunol Immunother. 2013:62:1249-60.

20. Yi $Y$, et al. The functional impairment of HCC-infiltrating gammadelta $T$ cells, partially mediated by regulatory T cells in a TGFbeta- and IL-10-dependent manner. J Hepatol. 2013;58:977-83.

21. Scurr M, et al. Highly prevalent colorectal cancer-infiltrating LAP(+) Foxp3(-) T cells exhibit more potent immunosuppressive activity than Foxp3(+) regulatory T cells. Mucosal Immunol. 2014;7:428-39.

22. Chen $L$, et al. Antitumor effect of malaria parasite infection in a murine Lewis lung cancer model through induction of innate and adaptive immunity. PLoS One. 2011;6:e24407.

23. Roetynck $S$, et al. Natural killer cells and malaria. Immunol Rev. 2006; 214:251-63.

24. Kalinski $\mathrm{P}$, et al. Natural killer-dendritic cell cross-talk in cancer immunotherapy. Expert Opin Biol Ther. 2005;5:1303-15.

25. Woan K, Reddy V. Potential therapeutic role of natural killer cells in cancer Expert Opin Biol Ther. 2007;7:17-29.

26. Ing R, Segura M, Thawani N, Tam M, Stevenson MM. Interaction of mouse dendritic cells and malaria-infected erythrocytes: uptake, maturation, and antigen presentation. J Immunol. 2006;176:441-50.

27. Corzo CA, et al. HIF-1alpha regulates function and differentiation of myeloid-derived suppressor cells in the tumor microenvironment. J Exp Med. 2010;207:2439-53.

28. Gros A, et al. Myeloid cells obtained from the blood but not from the tumor can suppress T-cell proliferation in patients with melanoma. Clin Cancer Res. 2012;18:5212-23.

29. Liu Y, et al. Cell surface receptor FPR2 promotes antitumor host defense by limiting M2 polarization of macrophages. Cancer Res. 2013;73:550-60. 
30. Ondondo B, Jones E, Godkin A, Gallimore A. Home sweet home: the tumor microenvironment as a haven for regulatory T cells. Front Immunol. 2013;4:197.

31. Mailloux AW, Young MR. Regulatory T-cell trafficking: from thymic development to tumor-induced immune suppression. Crit Rev Immunol. 2010;30:435-47

32. Kortylewski $M$, et al. Inhibiting Stat3 signaling in the hematopoietic system elicits multicomponent antitumor immunity. Nat Med. 2005;11:1314-21.

33. Kujawski M, et al. Stat3 mediates myeloid cell-dependent tumor angiogenesis in mice. J Clin Invest. 2008;118:3367-77.

34. Amin HM, et al. Selective inhibition of STAT3 induces apoptosis and G(1) cell cycle arrest in ALK-positive anaplastic large cell lymphoma. Oncogene. 2004;23:5426-34.

35. Tsareva SA, et al. Signal transducer and activator of transcription 3 activation promotes invasive growth of colon carcinomas through matrix metalloproteinase induction. Neoplasia. 2007:9:279-91.

36. Abad C, et al. Targeted STAT3 disruption in myeloid cells alters immunosuppressor cell abundance in a murine model of spontaneous medulloblastoma. J Leukoc Biol. 2014;95:357-67.

37. Ochoa AC, Zea AH, Hernandez C, Rodriguez PC. Arginase, prostaglandins, and myeloid-derived suppressor cells in renal cell carcinoma. Clin Cancer Res. 2007;13:721s-6s

38. Riley JL. PD-1 signaling in primary T cells. Immunol Rev. 2009;229:114-25.

39. Coppi A, et al. Heparan sulfate proteoglycans provide a signal to Plasmodium sporozoites to stop migrating and productively invade host cells. Cell Host Microbe. 2007;2:316-27.

40. Bindea G, Mlecnik B, Fridman WH, Pages F, Galon J. Natural immunity to cancer in humans. Curr Opin Immunol. 2010;22:215-22.

41. Boon $T$, et al. Identification of tumour rejection antigens recognized by $T$ lymphocytes. Cancer Surv. 1992;13:23-37.

42. DuPage $M$, et al. Endogenous $T$ cell responses to antigens expressed in lung adenocarcinomas delay malignant tumor progression. Cancer Cell. 2011;19:72-85

43. Galon J, et al. Type, density, and location of immune cells within human colorectal tumors predict clinical outcome. Science. 2006:313:1960-4.

44. Parmiani G, De Filippo A, Novellino L, Castelli C. Unique human tumor antigens: immunobiology and use in clinical trials. J Immunol. 2007;178:1975-9.

45. Bronte V, Zanovello P. Regulation of immune responses by L-arginine metabolism. Nat Rev Immunol. 2005:5:641-54.

46. Nagaraj S, Gabrilovich DI. Tumor escape mechanism governed by myeloidderived suppressor cells. Cancer Res. 2008:68:2561-3.

47. Mantovani A, Allavena P, Sica A, Balkwill F. Cancer-related inflammation. Nature. 2008;454:436-44.

48. Young MR, Wright MA, Pandit R. Myeloid differentiation treatment to diminish the presence of immune-suppressive CD34+ cells within human head and neck squamous cell carcinomas. J Immunol. 1997;159:990-6.

49. Kusmartsev SA, Kusmartseva IN, Afanasyev SG, Cherdyntseva NV. Immunosuppressive cells in bone marrow of patients with stomach cancer. Adv Exp Med Biol. 1998;451:189-94.

50. Kusmartsev SA, Ogreba VI. Suppressor activity of bone marrow and spleen cells in C57BI/6 mice during carcinogenesis induced by 7,12dimethylbenz(a)anthracene. Eksperimental'naia onkologiia. 1989;11:23-6.

51. Srivastava MK, et al. Targeting myeloid-derived suppressor cells augments antitumor activity against lung cancer. ImmunoTargets and therapy. 2012; 2012:7-12.

52. Lu T, et al. Tumor-infiltrating myeloid cells induce tumor cell resistance to cytotoxic T cells in mice. J Clin Invest. 2011;121:4015-29.

53. Sim SH, et al. Influence of chemotherapy on nitric oxide synthase, indoleamine-2,3-dioxygenase and CD124 expression in granulocytes and monocytes of non-small cell lung cancer. Cancer Sci. 2012;103:155-60.

54. Srivastava MK, et al. Myeloid suppressor cells and immune modulation in lung cancer. Immunotherapy. 2012:4:291-304.

55. Yu H, Pardoll D, Jove R. STATs in cancer inflammation and immunity: a leading role for STAT3. Nat Rev Cancer. 2009;9:798-809.

56. Gabrilovich DI, Ostrand-Rosenberg S, Bronte V. Coordinated regulation of myeloid cells by tumours. Nat Rev Immunol. 2012;12:253-68.

57. Ostrand-Rosenberg S. Myeloid-derived suppressor cells: more mechanisms for inhibiting antitumor immunity. Cancer Immunol Immunother. 2010;59: 1593-600.

58. Bronte V, Serafini P, Mazzoni A, Segal DM, Zanovello P. L-arginine metabolism in myeloid cells controls T-lymphocyte functions. Trends Immunol. 2003;24:302-6.
59. Wu G, Morris SM Jr. Arginine metabolism: nitric oxide and beyond. Biochem J. 1998:336 ( Pt 1:1-17.

60. Nagaraj S, Schrum AG, Cho HI, Celis E, Gabrilovich DI. Mechanism of T cell tolerance induced by myeloid-derived suppressor cells. J Immunol. 2010; 184:3106-16.

61. Jiang Y, Li Y, Zhu B. T-cell exhaustion in the tumor microenvironment. Cell Death Dis. 2015;6:e1792

62. Tsukumo SI, Yasutomo K. Regulation of CD8(+) T cells and antitumor immunity by notch signaling. Front Immunol. 2018;9:101.

63. Liu $\mathrm{Q}$, et al. Plasmodium parasite as an effective hepatocellular carcinoma antigen glypican-3 delivery vector. Oncotarget. 2017:8:24785-96.

64. Shin SC, Vanderberg JP, Terzakis JA. Direct infection of hepatocytes by sporozoites of Plasmodium berghei. J Protozool. 1982;29:448-54.

65. Regev-Rudzki N, et al. Cell-cell communication between malaria-infected red blood cells via exosome-like vesicles. Cell. 2013;153:1120-33.
Ready to submit your research? Choose BMC and benefit from:

- fast, convenient online submission

- thorough peer review by experienced researchers in your field

- rapid publication on acceptance

- support for research data, including large and complex data types

- gold Open Access which fosters wider collaboration and increased citations

- maximum visibility for your research: over $100 \mathrm{M}$ website views per year

At $\mathrm{BMC}$, research is always in progress.

Learn more biomedcentral.com/submissions 